\title{
Réflexions sur les surfaces et mesures des états de surface du bois
}

\author{
P. TRIBOULOT \\ I.N.R.A. - Station de Recherches sur la Qualité des Bois \\ Centre de Recherches forestières \\ Champenoux, F 54280 Seichamps
}

\begin{abstract}
Résumé
Les liaisons entre l'aspect structurel du bois et l'aptitude à un emploi donné passent par la mesure d'un certain nombre de critères de qualité des produits en bois. Dans ce cadre, nous développons ici une réflexion sur la mesure des paramètres caractéristiques des états de surface qui se heurte à des problèmes inhérents au matériau-bois : hétérogénéité de structure et faible densité entre autres.

Une méthodologie de mesure à partir d'un palpage mécanique est proposée, ainsi que l'interprétation des résultats obtenus par cette méthode.
\end{abstract}

D'importants programmes de recherches sont actuellement en cours, notamment en France, pour connaître, à partir de tests non destructifs, l'influence des diverses sources biologiques de variation (sol, climat, sylviculture, hérédité) sur la qualité du bois produit. L'objectif final est de pouvoir définir le matériel végétal et les conditions de cuIture qui permettent à la forêt de fournir à l'industrie nationale la matière première la plus à même de satisfaire ses besoins.

Cependant, s'il est logique de penser que les propriétés physiques étudiées : densité, homogénéité et rétractibilité notamment exercent une influence directe sur la qualité des produits industriels, encore convient-il de le vérifier expérimentalement et d'évaluer de façon chiffrée l'incidence au niveau de la production d'un gain sur tel ou tel paramètre mesuré au laboratoire.

Un critère important d'appréciation des produits finis ou semi-finis en bois (placages tranchés ou déroulés, meubles, ...) est l'état de surface qui peut intervenir de façon directe comme argument de vente en liaison avec les propriétés esthétiques ou tactiles de l'objet fabriqué, mais qui exerce également une influence vitale sur :

- la ténacité des joints collés (EBEwele et al., 1980);

- la mouillabilité des revêtements superficiels (laquage, peintures, vernis, ...) ;

- le frottement des pièces fonctionnelles et d'adhérence;

- la prise au feu des éléments en bois (C.T.B., 1973), 
et bien entendu, sur tous les aspects du jugement «objectif » de la qualité d'un usinage, d'un traitement de surface pour une essence donnée. Il se pose alors le problème du contrôle et de la mesure des états de surface en bois.

L'aspect général du problème posé se décompose en trois niveaux : née ;

- la spécification d'un état géométrique d'une surface pour une fonction don-

- les moyens d'élaboration de l'état de surface spécifié;

- le contrôle et la mesure qui permettent de vérifier si une surface correspond bien à la spécification énoncée.

Nous aborderons sommaircment les deux premiers niveaux et développerons le troisième qui représente à lui seul un vaste domaine encore trop peu exploré dans les sciences du bois, et qui en l'absence de critères qualitatifs reste subjectif et toujours sujet à discussions.

\section{Définition d'une surface}

D’une façon générale, une surface peut être définie comme la partic la plus à gauche du diagramme d'équilibre des phases d'un corps pur. Mais dans le cas du bois, une élévation de température ne conduit pas à la liquéfaction, ni à la vaporisation de celui-ci, mais à la décomposition des constituants. Aussi, parlerons-nous de la «frontière » entre l'état solide du bois et l'état gazeux ou liquide de l'environnement. D'un point de vue mathématique, une surface peut être décomposée en fonctions polynomiales, mais pour le bois, un certain nombre de profils représentatifs des surfaces sont extrêmement complexes à définir (fig. 1) (FAyolle, 1983), et pourtant fréquemment rencontrés (zone de vaisseaux, arrachement de fibres ou paquets de fibres).

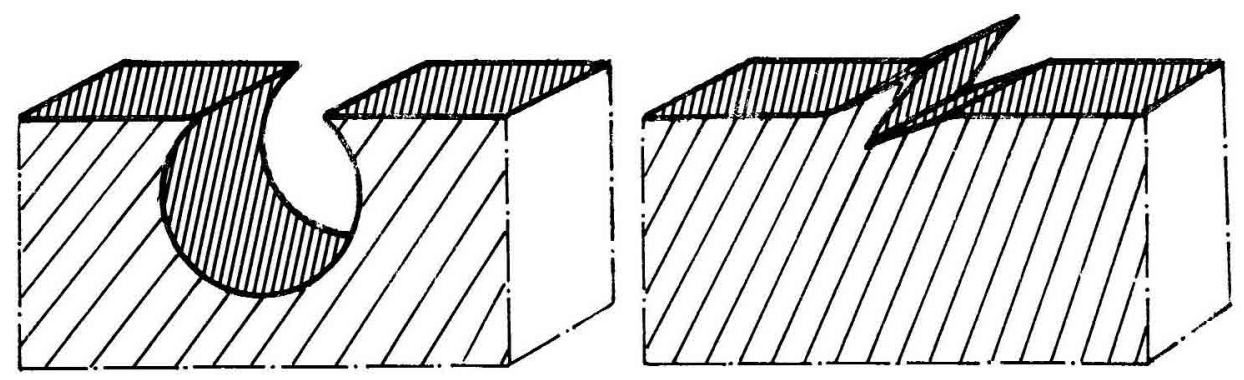

FIG. 1

Profils représentatifs de surfaces complexes.

Typical profile of complexe surfaces.

Il faut cependant introduire la notion d'échelle dans la mesure, et considẻrer les dimensions des éléments anatomiques du bois. La surface se définit alors par 
ÉTATS DE SURFACE DU BOIS

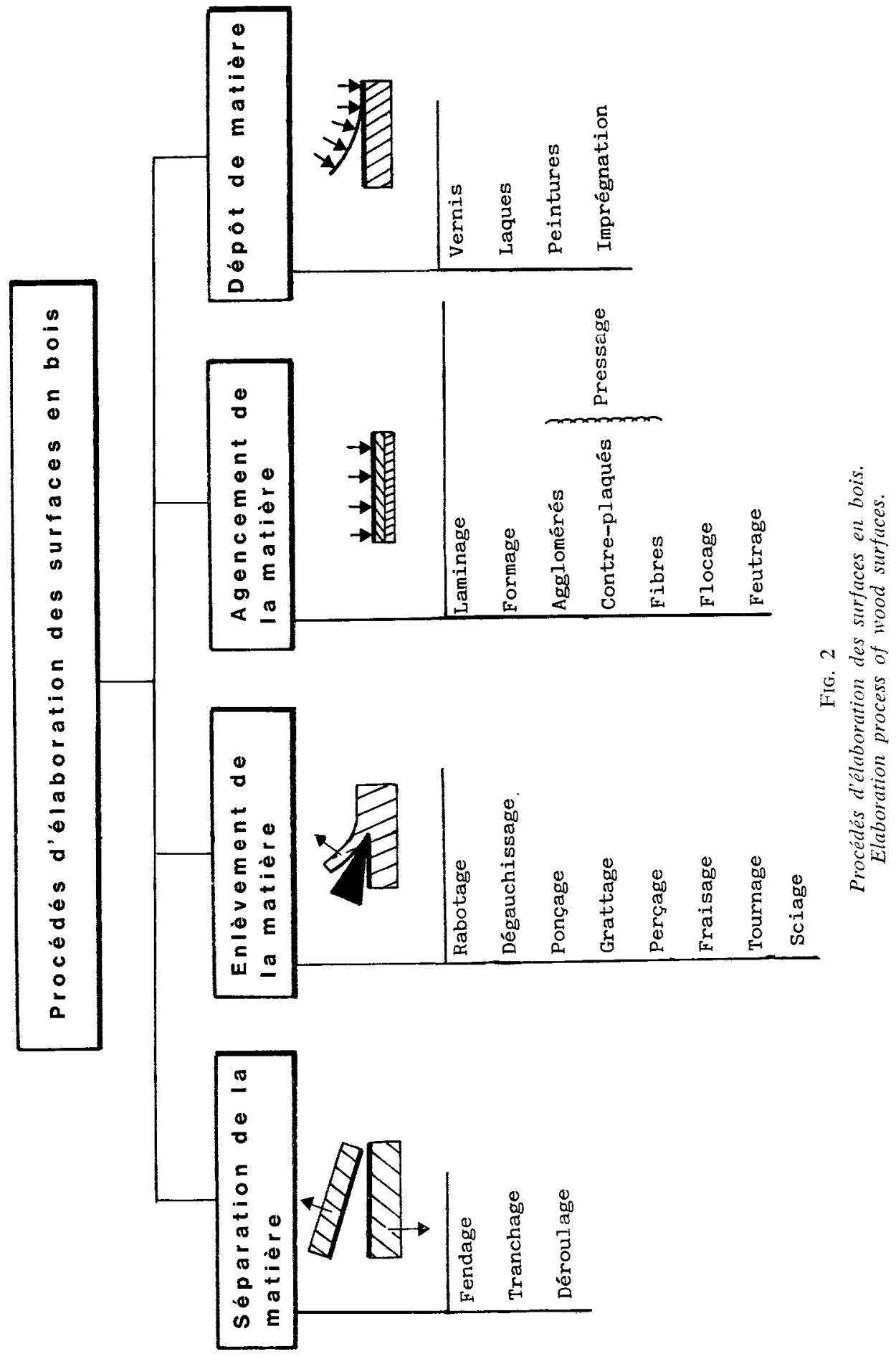


rapport au niveau des dimensions minimales des éléments que l'on veut apprécier [microfibrilles, fibres (10 microns), trachéides (20 microns), vaisseaux (200-500 microns)].

\section{Procédés d'élaboration et spécification}

Les procédés d'élaboration des surfaces en bois sont fort variés. Pour chacun de ces procédés, la microgéométrie obtenue dépend de nombrcux paramètres d'usinage. Il s'ensuit une grande variété dans les élats de surface pouvant être obtenus, d'autant que chaque essence, de par son plan ligneux, multiplic cncore pour chaque type d'usinage ces états de surface. On peut cependant résumer les procédés d'élaboration selon le schéma de la figure 2 .

Parmi les paramètres d'usinage susceptibles d'avoir une influence sur l'état de surface, nous pouvons retenir, outre l'essence usinée :

- le régime de coupe (vitesse de coupe, profondeur de passe, avance, ...);

- la géométrie de l'outil de coupe;

- la qualité de l'arête tranchante et l'état de la face d'attaque ;

- l'usure de l'outil ;

- la température;

- la rigidité des éléments des machines d'usinage;

- la présence d'une barre de pression (déroulage).

Quant aux spécifications des états de surface obtenus par les différents procédés énumérés précédemment, elles n'existent pas en tant que normes et il apparaît là une grande lacune qui reste à combler.

\section{3. État de surface}

Nous nous limiterons dans cette étude à l'état géométrique de la surface, car il correspond à l'état de surface technologique; l'état physico-chimique de celle-ci n'est pas abordé (il nécessite la mise en cuvre de techniques d'analyse par diffraction et spectroscopie).

\subsection{Terminologie générale}

Une surface peut être a priori caractérisée par un profil représentatif. Prenons l'exemple d'une feuille de placage tranchée ou déroulée (fig. 3).

La séparation et le tracé de ces divers profils peuvent être obtenus par les appareils de mesure (voir paragraphe 3.3.).

Signalons que l'étude descriptive d'un état de surface ne peut se contenter, et $a$ fortiori dans le cas du bois, de l'étude d'un profil unique. Le relevé d'un ensemble de profils apporte un nombre important de renseignements complémentaires sur l'hétórogénéité et l'anisotropie des surfaces. Dans les cas d'anisotropie de surface, les normes (des matériaux pour lesquels la norme existe) précisent que la direction de mesure doit être celle qui donne la valeur maximale de l'écart géométrique considéré (AFNOR, 1972) (fig. 4). 


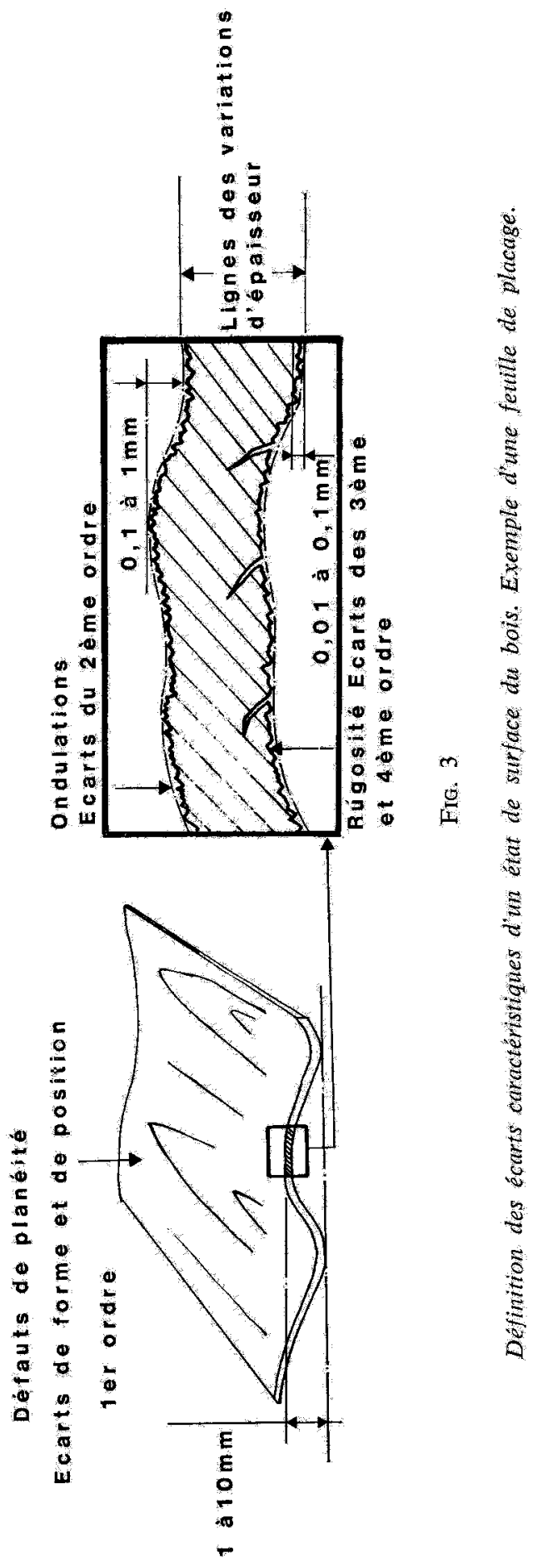



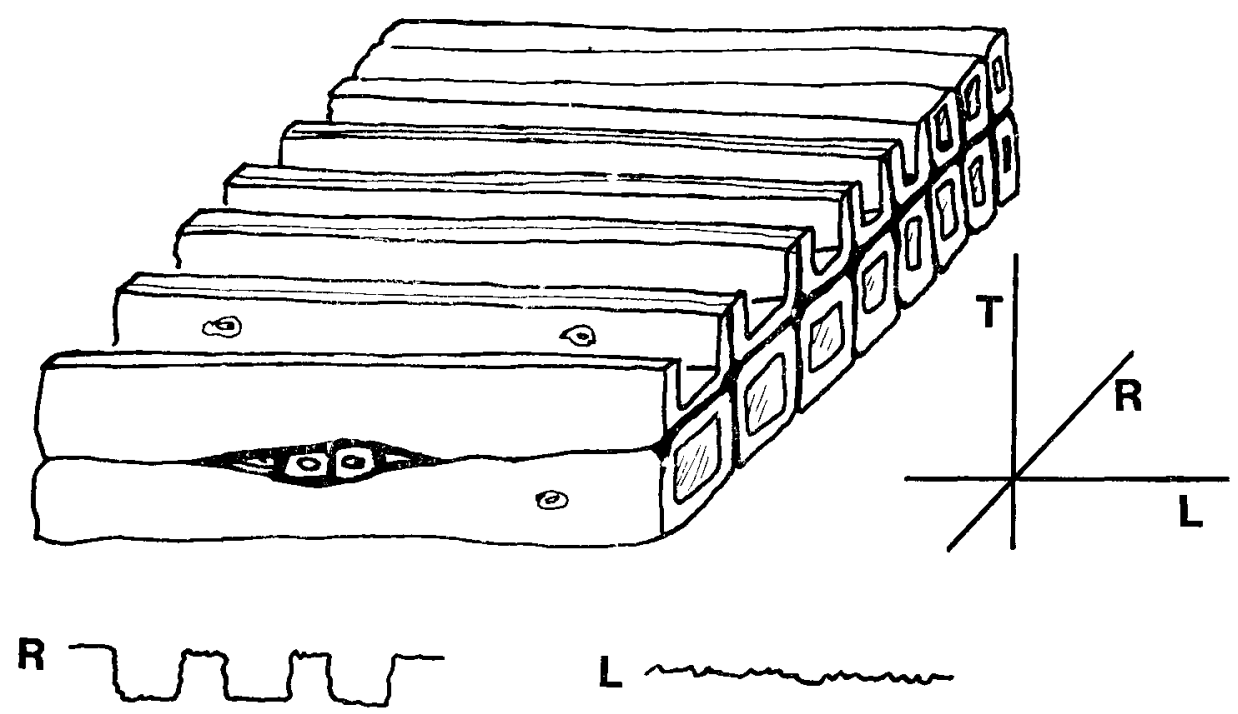

FIG, 4

Anisotropie de l'état de surface du bois.

Roughess anisotropy.

Enfin, il est utile de préciser que le terme état de surface englobe les défauts des $1^{\prime r s}, 2^{\prime \prime s}, 3^{n s}$ et $4^{\prime \prime \prime}$ ordres et que le terme rugosité s'applique uniquement aux $3^{n s}$ et $4^{\text {es }}$ urdres.

\subsection{Critères classiques d'état de surface}

Nous ne reprenons pas ici en détail l'inventaire des différents critères de profil défini dans les normes AFNOR (AFNOR, 1972) par exemple. En effet, il apparaît rapidement qu'aucun des critères n'est satisfaisant pour le bois, et surtoul qu'aucun d'entre eux n'est suffisant pour caractériser entièrement un profil (même dans le cas de surfaces isotropes et de matériaux homogènes). Pour mémoire, les plus courants de ces critères sont explicités sur la figure 5 :

avec $\operatorname{Rp}=\frac{1}{\mathrm{~L}} \int_{0}^{\mathrm{I}} \mathrm{Z} \mathrm{dX}$

On peut noter également le critère Ra ou «écart moyen arithmétique de la rugosité par rapport à la ligne moyenne », qui est le critère le plus utilisé et défini par :

$$
\mathrm{Ra}=\frac{1}{\mathrm{~L}} \int_{n}^{\mathrm{L}}|\mathrm{Z}-\mathrm{Rp}| \mathrm{dX} \text {; l'cxemple suivant illustre la limitation de ces cri- }
$$
tères. 


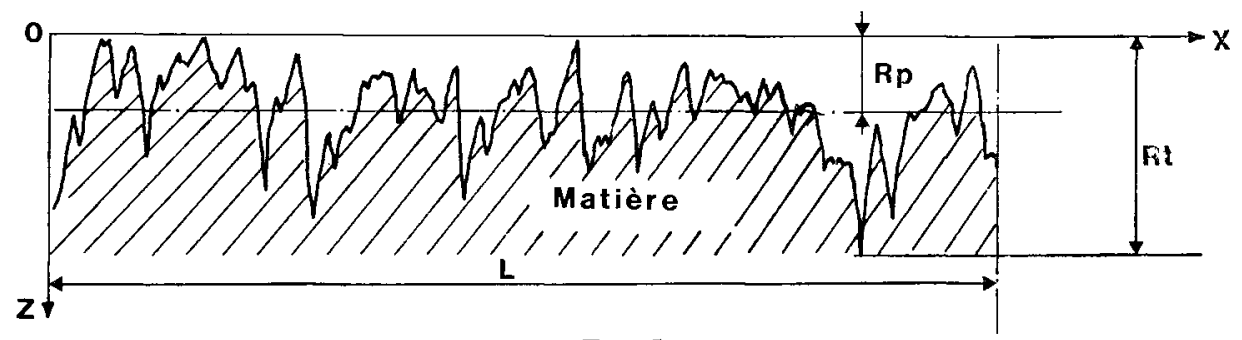

Fig. 5

Critères classiques d'état de surface des matériaux isotropes.

Roughness classical criteria of isotropic materials.

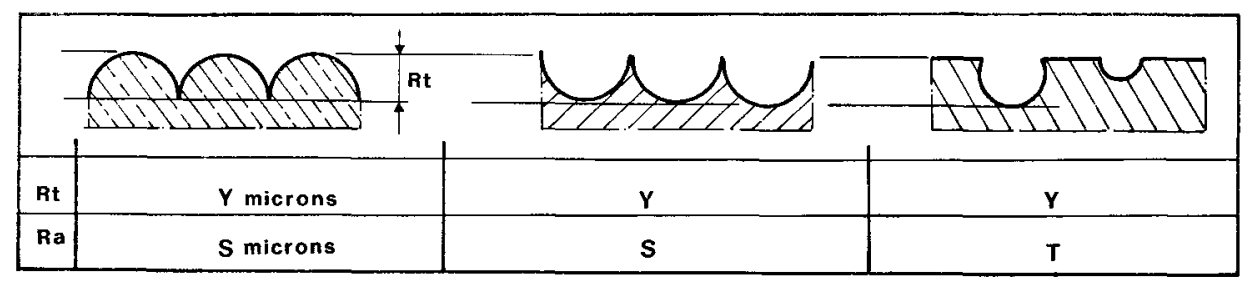

FIG. 6

Limitation des critères classiques d'état de surface.

Limited condition of roughness classical criteria.

\subsection{Inventaire des méthodes de mesure}

Pour plus de détails sur les procédés de mesure des états de surface, on pourra se référer aux ouvrages de Riout (1979). Parmi ces procédés, un certain nombre d'entre eux ont été appliqués au bois; une étude bibliographique récente dresse cet inventaire, fait une analyse critique des mesures déjà réalisées dans certains laboratoires et présente les critères classiques d'état de surface appliqués au bois (MARCHAL, 1983).

On peut cependant retenir les différentes familles de procédés d'exp'oration des surfaces suivantes :

- comparaison visotactile avec des échantillons;

- exploration par rugosimètres mécaniques (reproduction d'empreintes par pressage ; appareil de l'abbé CAYERE) ;

- exploration par méthodes pneumatiques;

- exploration par méthodes capacitives;

- méthodes optiques ;

- exploration par palpage mécanique et traitement électronique et informatique.

Parmi ces dispositifs, les dispositifs par palpage sont ceux mis en œuvre plus rapidement, d'une façon automatique, d'une grande précision, et qui pour l'instant paraissent les plus adaptés au bois. 


\subsection{Méthode de mesure des états de surface par palpage}

Cette méthode de mesure comporte un palpeur mécanique dont l'aiguille exploratrice parcourt un profil à étudier. Les déplacements verticaux du palpeur produisent dans un circuit électrique approprié des variations de tension pouvant être amplifiées et enregistrées. De récents perfectionnements ont été apportés à cette technique (MrGNOT, 1983 ; MATHIA, 1983) et permettent l'étude d'un profil et également l'étude d'une surface. La figure 7 illustre ce dispositif.

Sans entrer dans les détails techniques, le dispositif a l'avantage d'être extrêmement souple d'emploi et d'accéder à l'état géométrique d'une surface réelle pouvant être définie par les hauteurs $Z(X, Y)$. De plus, on peut effectuer tous les calculs souhaités pour caractériser cet état ; il convient alors d'étudier cette hauteur $Z$ comme une variable aléatoire en utilisant les traitements statistiques appropriés et développés actuellement dans certains laboratoires (voir paragraphe 3.6.).

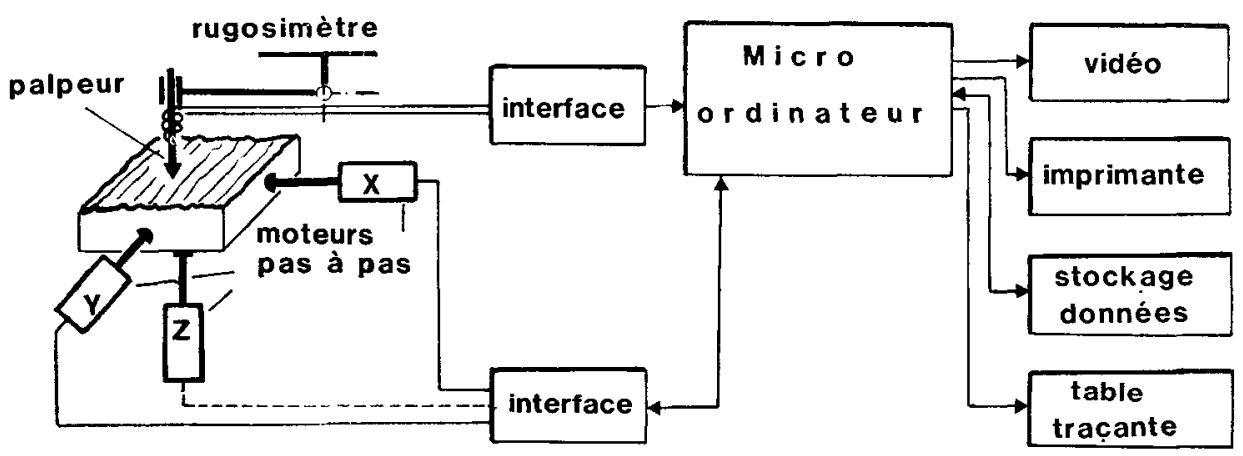

FIG. 7

Dispositif expérimental de mesure des états de surface.

Experimental device for roughness measurement.

\subsection{Problèmes inhérents au bois}

\subsection{Aspect photographique des profils à explorer}

Nous abordons cet aspect du problème par une étude des profils photographiques obtenus sur des copeaux de bois déroulés, tranchés, et sur du bois massif microtomé (dans le plan RT), de deux essences caractéristiques : le douglas et le chêne.

Nous nous trouvons confronté à un problème de mesure d'état de surface très particulier. Les éléments anatomiques du matériau, en particulier cas des feuillus à zones de vaisseaux très marqués, sont prédominants sur l'état de surface obtenu par rapport aux irrégularités de surface liées au processus d'obtention de celle-ci. Aussi, nous devons pouvoir séparer "l'action" de l'anatomie sur l'état de surface obtenu afin de pouvoir, par exemple, apprécier un type d'usinage ou un réglage de machineoutil, où nous devons cumuler les deux effets pour apprécier la qualité du produit fini. 


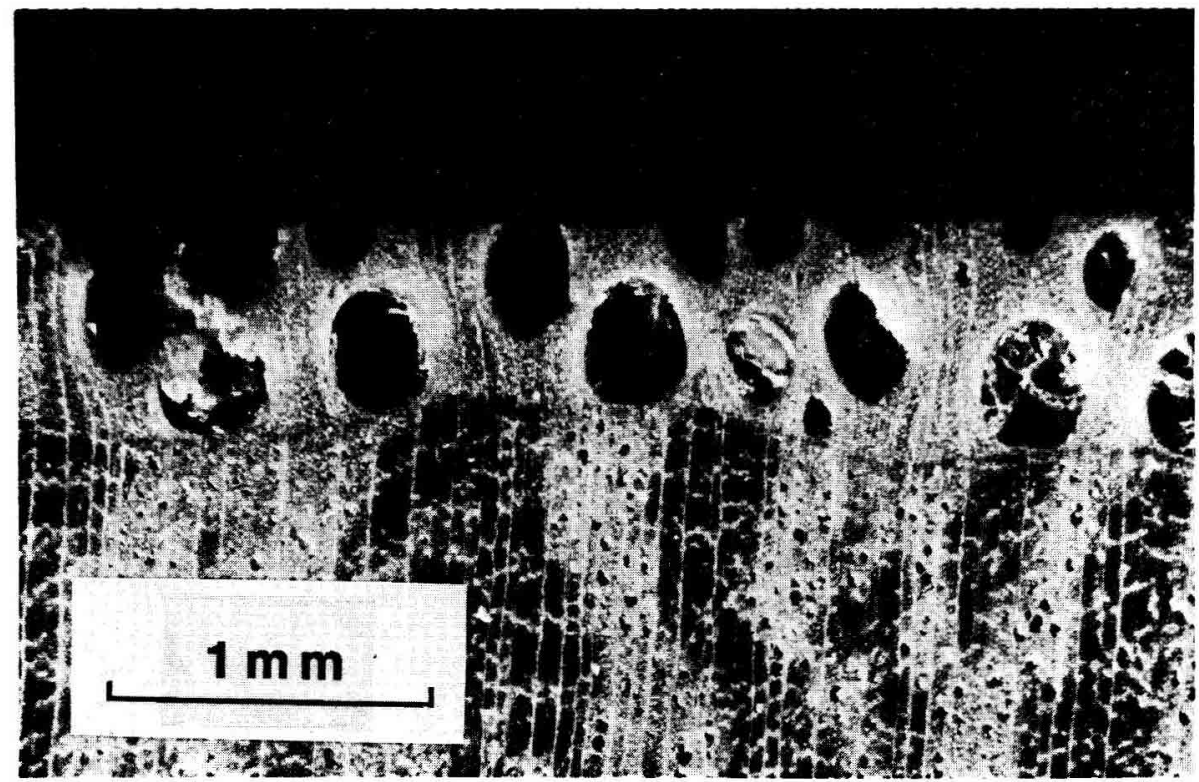

Photographie 1

Profil photographique obtenu sur du chêne dans la zone de vaisseanx (surface microtoméc). Plaotographic profil of oak in the vessel zone (after micro-cutting).

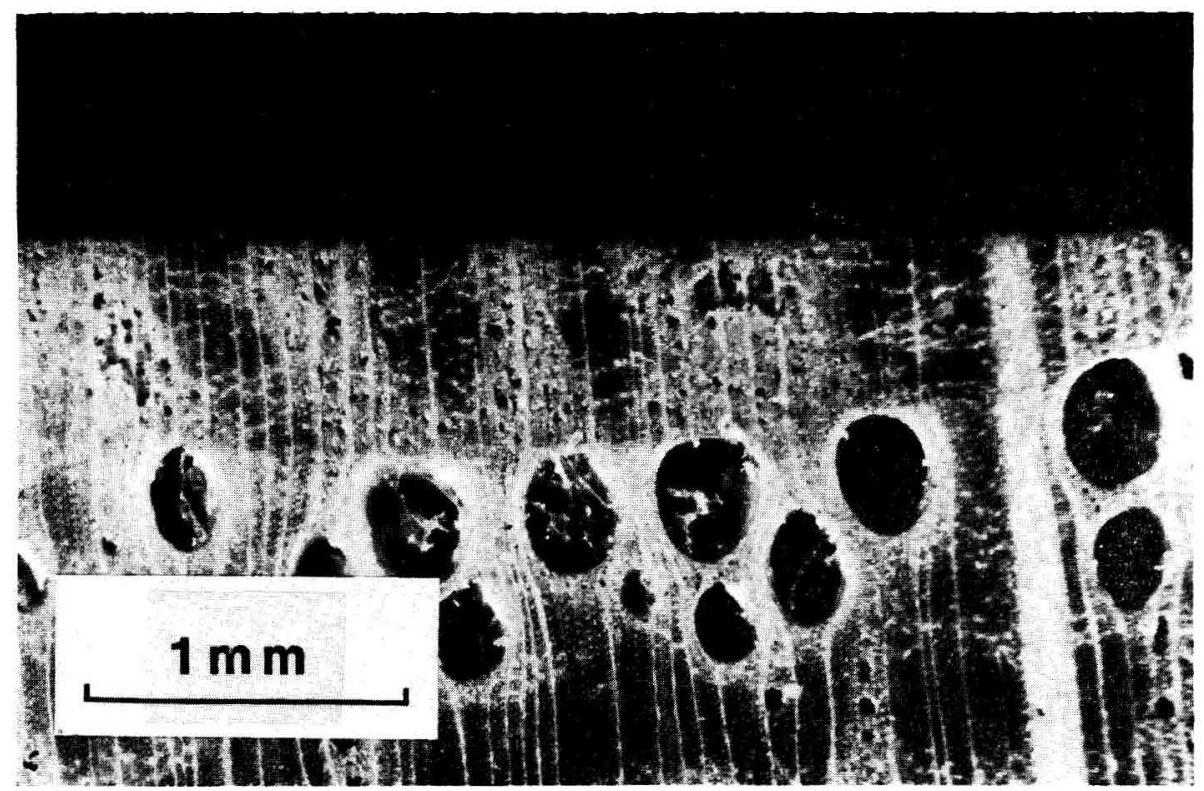

PhotogRaphie 2

Profil photographique obtenu sur du chêne en dehors de la zome de vaisseani (surface microtomée).

Photographic profil of oak outside the vessel zone (after micro-cutting). 

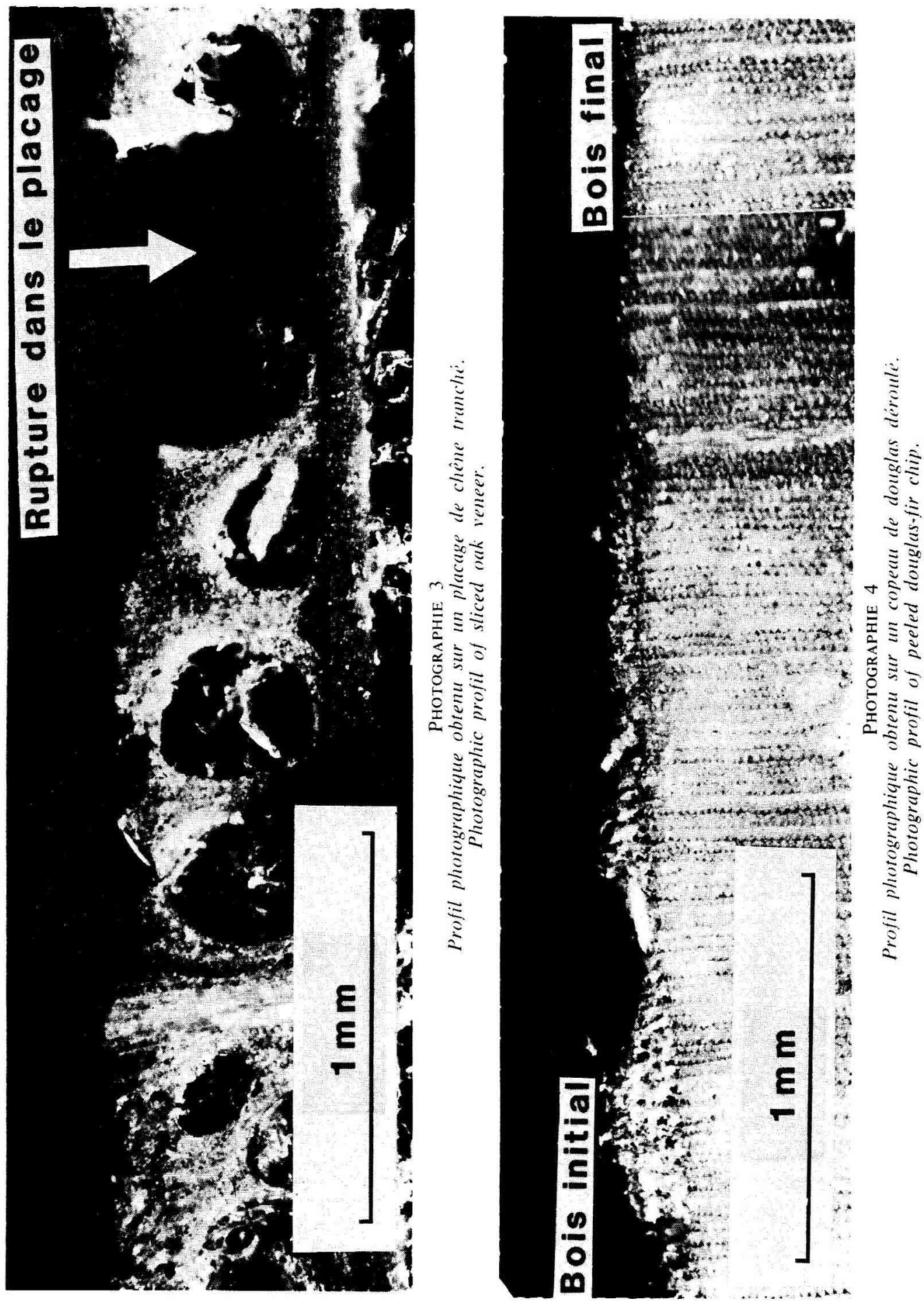


\subsection{Influence du palpeur sur les mesures}

Le palpeur doit être en mesure d'explorer parfaitement un profil sans en altérer la géométrie. Il est illusoire dans le cas du bois d'accéder au profil réel du matériau sur des surfaces telles que celles définies à la figure 1. D'autre part, le diamètre en pointe du palpeur occasionne un filtrage mécanique du profil réel, facilement mis en évidence sur la figure 8 .

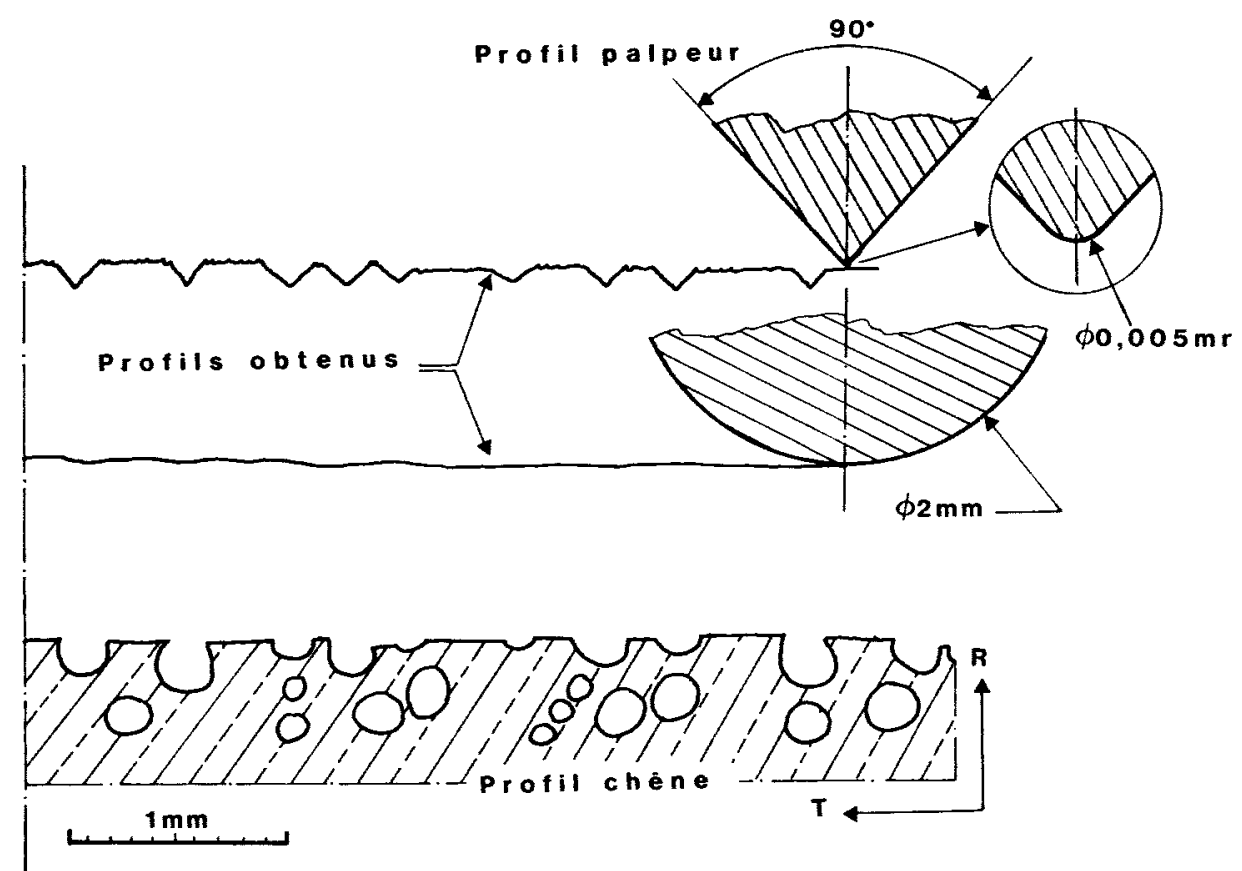

Fik. 8

Filtrage mécanique d"un profil réel dû au profil du palpeur.

Mechanical filtration of a real profile generated by the transducer.

Enfin, nous avons pensé qu il était nécessaire d'apprécier les déformations liécs au contact palpeur/bois. Pour ce faire, nous utilisons the approche classique de résistance des matériaux traitant du contact entre surfaces (Tımoshenko, 1968). Les hypothèses que nous utilisons sont extrêmement simplificatrices :

- déformation du palpeur négligeable;

- rayons de courbure connus au point de contact;

- corps parfaitement élastiques, homogènes et isotropes dans la dircetion de sollicitation ;

- pressions de contact perpendiculaires au plan de contact ; tement.

- charge normale; on néglige l'influence des forces tangenticlles dues au frot- 
On aboutit alors pour un contact bille sur suiface en bois à une expression du type :

$$
i=0,77 \quad \int^{3} \frac{2 \mathrm{p}}{\mathrm{d}_{1}}\left(\frac{1}{\mathrm{E}_{1}} \therefore \frac{-1}{\mathrm{E}_{2}}\right)^{\ddot{*}}
$$

avec $i=$ enfoncement du palpeur dans le bois;

$\mathbf{P}=$ charge appliquéc:

$\mathrm{d}_{1}=$ diamètre de pointe du palpeur ;

$\mathrm{E}_{1}=$ module d'Young du palpour :

$\mathbf{E}_{2 .}=$ module d'Young du bois (direction de charge).

avec $E_{1}$ très supéricur à $E_{2}$. la déformation résultante du contact se linite alors à l'enfoncement $\lambda$ du palpeur dans le bois.

La figure 9 donne les résultats de ce calcul pour différents niveaux de charge.

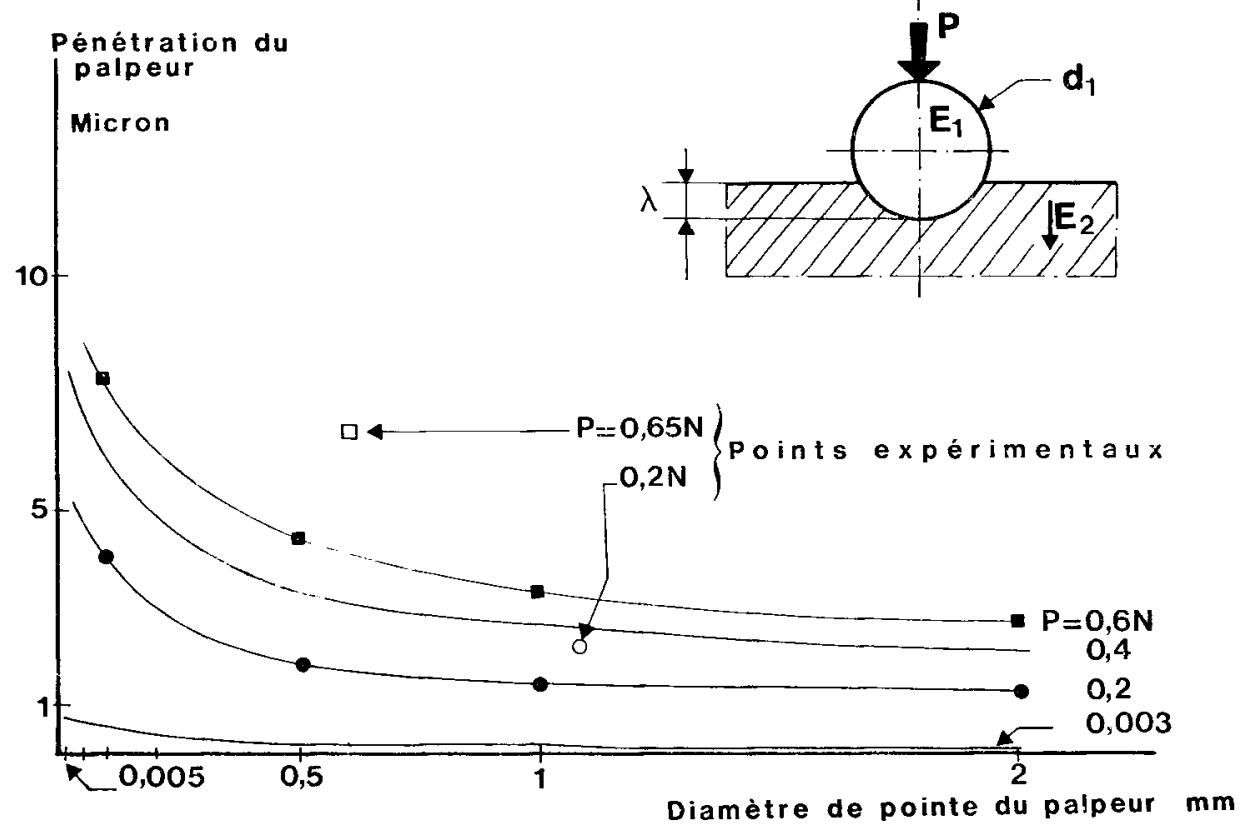

Firi, y

Pénétration de palperer dans le bois en fonction da diamètre de pointe et pour differents niveratex de charge'.

Indentation of the transducer in wood as a function of the dianeter of the head for sereral load levels.

(Points expérimentaux KiKala, 1958). ( $\left.\mathrm{F}_{2}=\mathrm{E}_{1:}=2000 \mathrm{MPa}\right)$.

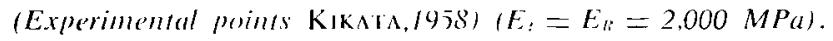


Le niveau de charge le plus bas sur la figure 9 correspond à un dispositif commercial de palpage pouvant être intégré dans la chaîne de mesure décrite à la figure 7 et possédant un capteur standard conique de diamètre de pointe égal à 5 microns. L'effet de pénétration qui en résulte dans le bois (inféricur au micron) peut être négligé. Les essais sur un bois hétérogène peuvent conduire à des erreurs d'interprétation importantes si l'on ne respecte pas certaines précautions liées essentiellement à la charge appliquée au palpeur. Sur l'exemple de la figure 10 , on a représenté les variations de densité du douglas sur trois comes et "l'état de surface " mesuré après rabotage; nous avons utilisé un dispositif dont les caractéristiques ćtaicnt les suivantes :

- diamètre de pointe du palpeur : $2 \mathrm{~mm}$;

- charge appliquée : $0.5 \mathrm{~N}$.
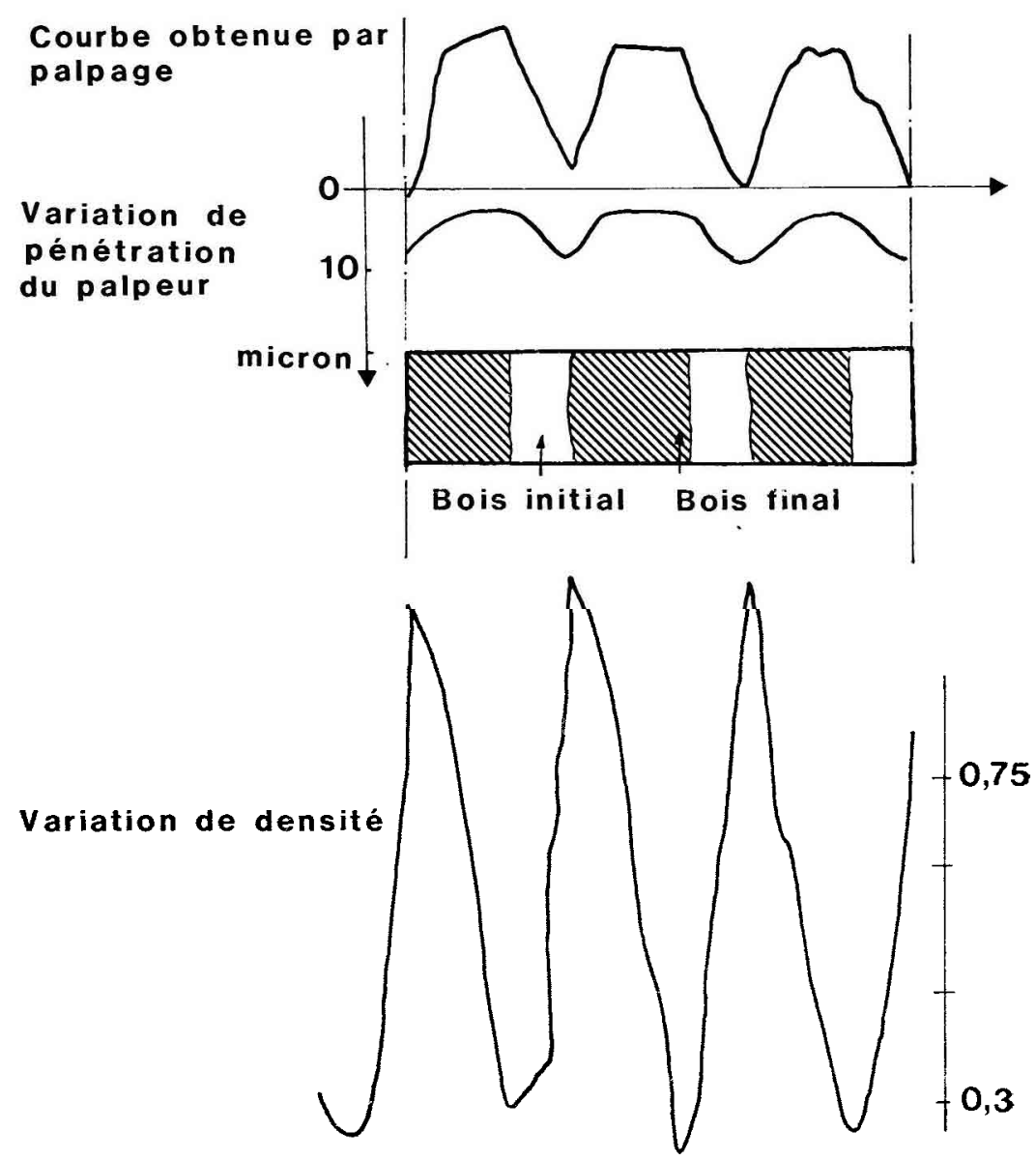

FIG. 10

Variation de pénétration du patpeur

lors da passage bois initial - bois final sur douglas massif.

Penetration of the head of transducer in early and late wood douglas fir. 
Sur la figure 10 est représentée également la courbe des variations de pénétration du palpeur le long du profil considéré ; cette courbe est calculée à l'aide de l'expression (1) en utilisant les relations classiques entre densité et module d'Young du bois (pour un taux d'humidité donné).

On peut constater ici que l'on a mesuré à la fois un "état de surface », et également une variation de microdureté sur le bois considéré. Il résulte de nos expériences que le bon choix pour une mesure de rugosité de surface du bois se trouve être:

- palpeur conique d'angle 90". diamètre de pointe $10-20 \mu$, ce qui minimise le filtrage mécanique du profil sans risque d'accrochage;

- charge appliquée de l'ordre de $0.003 \mathrm{~N}$; ce niveau de charge est obtenu sur certains appareils commercialisés; il permet un contact permanent avec la surface pour une vitesse de palpage de l'ordre de $1 \mathrm{~mm} /$ seconde et un endommagement quasi nul.

La figure 11 montre, d'une part un profil photographique obtenu sur du chêne massif, et d'autre part l'enregistrement effectué avec un palpeur du même type que celui décrit précédemment.

(B)
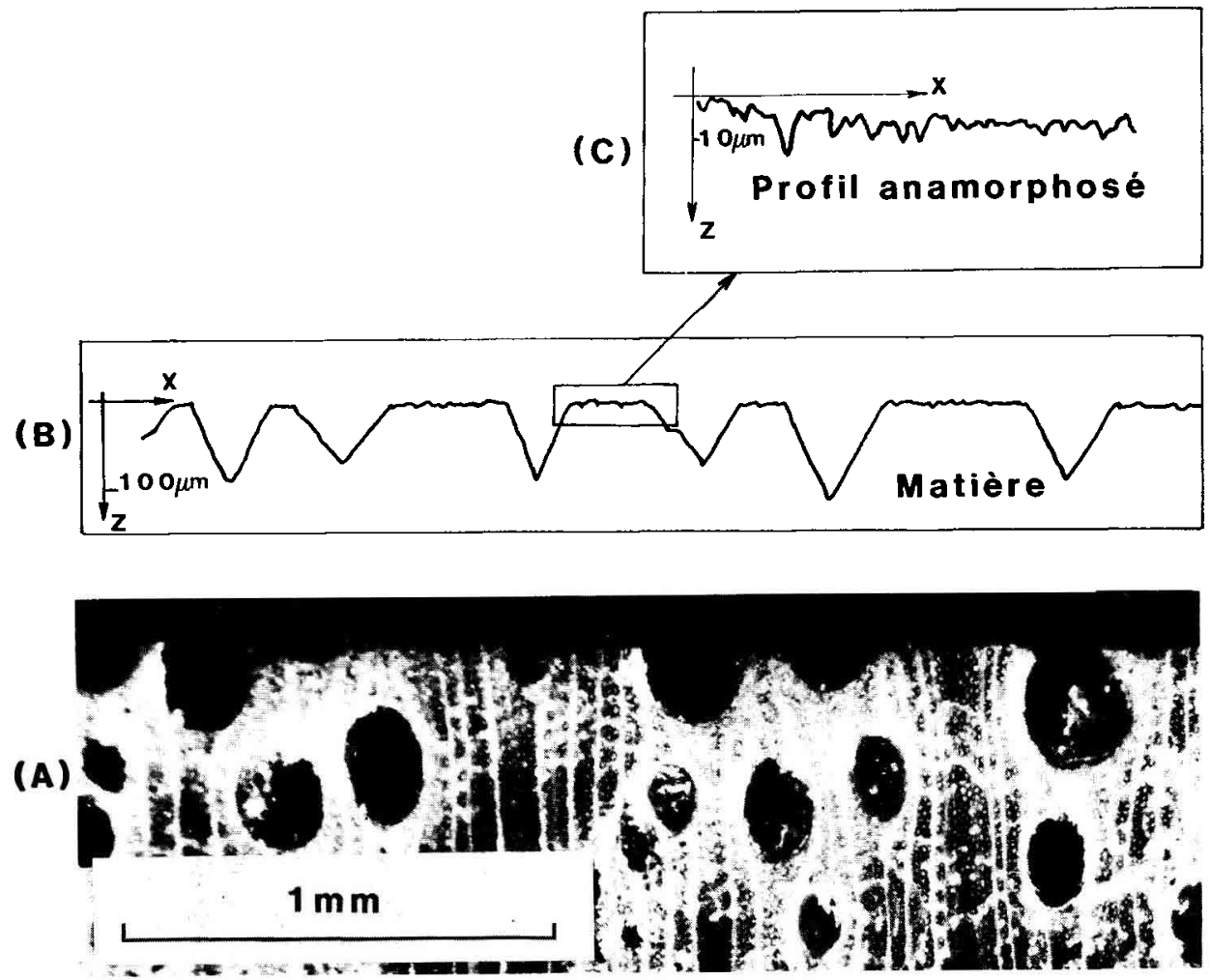

FIG. 11

Profil photographique et enregistrement des profils de rugosité d'une feuille de placage de chêne tranché.

Photographic profile and roughness of sliced oak veneer. 
La figure 12 présente les enregistrements obtenus sur douglas dans les zones respectives de bois initial et de bois final.
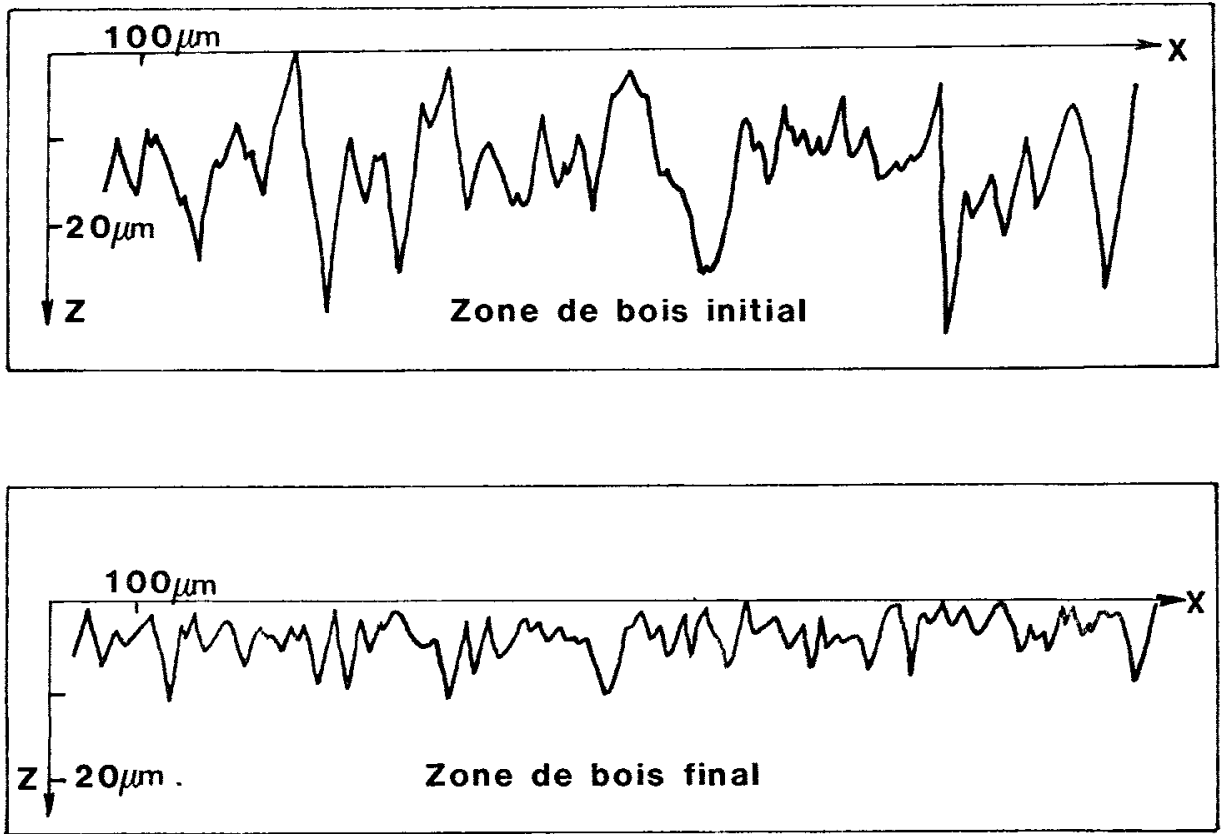

F16. 12

Profils de rugosité d'un copeau de douglas déroule dans les zones de bois initial et de bois final.

Douglas-fir chip roughess peeded in early and late wood.

3.6. Analyse des profils obtenus par palpage

Nous ne reviendrons pas ici sur le calcul et l'utilisation des critères classiques d'état de surface qui ont montré leurs limites et nous n'aborderons que l'aspect statistique de l'analyse des profils à partir d'un dispositif tel que celui du paragraphe 3.4. Les essais ont été effectués au laboratoire du Professeur Mignor (1983, op. cit.) : palpeur conique diamètre de pointe $5 \mu$, force du stylet $0,003 \mathrm{~N}$. La première série d'essais porte sur un échantillon de chêne tranché. La figure 13 représente le profil initial issu de l'appareillage et portant sur 1000 points $Z(X)$. On retrouve sur cet enregistrement les «vallées» caractéristiques de la présence des vaisseaux (courbe A) : direction du palpage tangentiel aux cernes annucls.

Sur cet essai, la courbe (B) représente un lissage mathématique du profil initial qui peut être assimilé ici, en première approche, à la rugosité inhérente à la structure 
du chêne (présence de gros vaisseaux). Le calculateur effectuc alors la différence des deux profils et restitue la courbe (C) qui peut être assimilée à la rugosité inhérente au procédé de tranchage pour le chêne expérimenté.
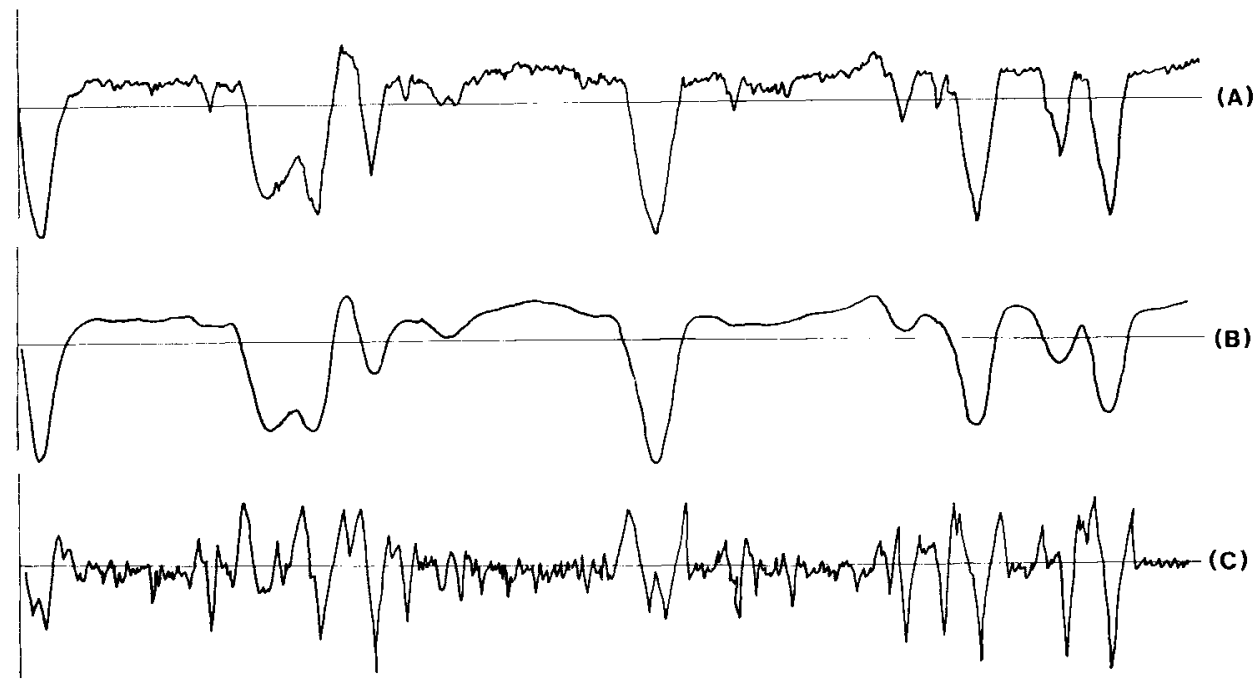

Fro. 13

Profils d'atat de surface obtenus stor ane jeatle de placage de chime tranchés.

Ronghness profile of sliced oak veneer.

(A) profil initial - initial profile.

(B) lissage du profil initial ou rugosité induite par la présence des vaisseatux - smooth intiall profile or roughloness generated by the vessels.

$(C)=(A)-(B)$. Le profil amplifié est caractéristique de lopération de tranchage sur chêne-Amplified profile is typical for slicing of oak.

A partir des données $Z(X)$ stockées dans le calculateur ou sur disquettes, l'analyse statistique peut s'effectuer. Nous ne retiendrons ici que les paramètres les plus intéressants :

- l'écart type $\ell^{\prime}$ (ou la variance $\sigma^{*}$ ) de la fonction de distribution des hauteurs

$$
\ell_{.}=\sigma^{2}=\int_{-\infty}^{+\infty}(Z-Z)^{-2} \cdot P(Z) \cdot d Z
$$

où $\mathrm{P}(\mathrm{Z})$ est la densité de distribution des hauteurs.

- le paramètre d'obliquité ou « Skewness », $\mathrm{S}_{\mathrm{k}}$ :

$$
S_{k}=\frac{l_{3}}{\left(u_{2}\right)^{* / 2}}=\frac{l_{33}}{\sigma^{3}}
$$

avec $\iota_{i}=$ moment centré d'ordre $\mathrm{i}=\int_{-\infty}^{+\infty}(Z-\bar{Z})^{\mathrm{i}} \cdot \mathrm{P}(\mathrm{Z}) \cdot \mathrm{d} Z$ 
- le paramètre d'aplatissement ou «Curtosis», $E_{k}$ :

- la fonction d'autocorrélation.

$$
E_{k}=\frac{1 l_{1}}{(! n)^{4+2}}
$$

Physiquement, si :

$\mathrm{S}_{\mathrm{k}}=0$, la courbe de densité des hauteurs est symétrique : le profil l'est également. $\mathrm{S}_{\mathrm{k}}<0$, le profil est plein; il y a concentration de matière dans la partie supérieure du profil.

$\mathrm{S}_{\mathrm{k}}>0$, le profil est creux; voir tableau récapitulatif no I (Rrour, 1979).

$\mathrm{E}_{\mathrm{k}}=3$, la distribution est normale (gaussienne).

$\mathrm{E}_{\mathrm{k}}<3$, la répartition est aplatie.

$\mathrm{E}_{\mathrm{k}}>3$, la répartition est pointue. surface.

Ces deux paramètres $S_{k}$ et $E_{k}$ caractérisent l'étalement vertical d'un profil de

La fonction d'autocorrélation caractérise l'étalement horizontal du profil et permet en outre d'apprécier comment la hauteur d'un point du profil est influencée par la hauteur du même profil en un point situé à une distance $\beta$, done de détecter les éventuelles périodicités. Ia fonction d’autocorrélation s'écrit sous la forme :

$$
R(\beta)=\frac{1}{\sigma^{2}(L-\beta)} \int_{1}^{L} Z(X) \cdot Z(X+\beta) d X
$$

un profil aléatoire pur comme en général dans le cas du bois (feuillus à zones poreuses) présentera une fonction d'autocorrélation exponentielle.

Sur l'exemple traité en début de paragraphe, les résultats ont élé les suivants (référés à la courbe $\mathrm{C}$ ) : voir tableau 2 .

\subsection{Développenents du principe}

\subsection{Aspect tridimensionnel}

Dans le paragraphe précédent, nous nous sommes limité à l'analyse d'un profil sensé être représentatif de toute la surface; or nous avons vu (paragraphe 3.1.) que les surfaces en bois pouvaient présenter un fort degré d'anisotropie. La méthodologie utilisée sur un profil peut être étendue à toute la surface par palpages successifs parallèles en incrémentant le déplacement du moteur $Y$ (voir fig. 7). On accède alors à une représentation tridimensionnelle des surfaces, et l'analyse statistique peut se faire sur les points enregistrés. Cette étude de surface permet d'obtenir deux critères extrêmement intéressants : la surface portante ou taux de surface de portance, et la surface développée. Le taux de portance est égal à l'aire de la surface plane, intersection du plan d'équation $\mathrm{Z}=\mathrm{C}$ avec la surface explorée; ce critère serait utile pour toutes les études relatives au collage des bois et à la mouillabilité. La surface développée, surtout dans le cas des feuilles à gros vaisseaux, apporterait des éléments pour tous les problèmes de revêtements de surface. 


\section{TABILAU 1}

Tableau récapitulatif des profils curactéristiques d'états de surface.

courhes de densité correspondantes et valeurs des parametres d'obliquité et daplatissement.

Recapitulatory table of typical profile of roughness,

density corresponding curves and shewness and curtosis parametors.

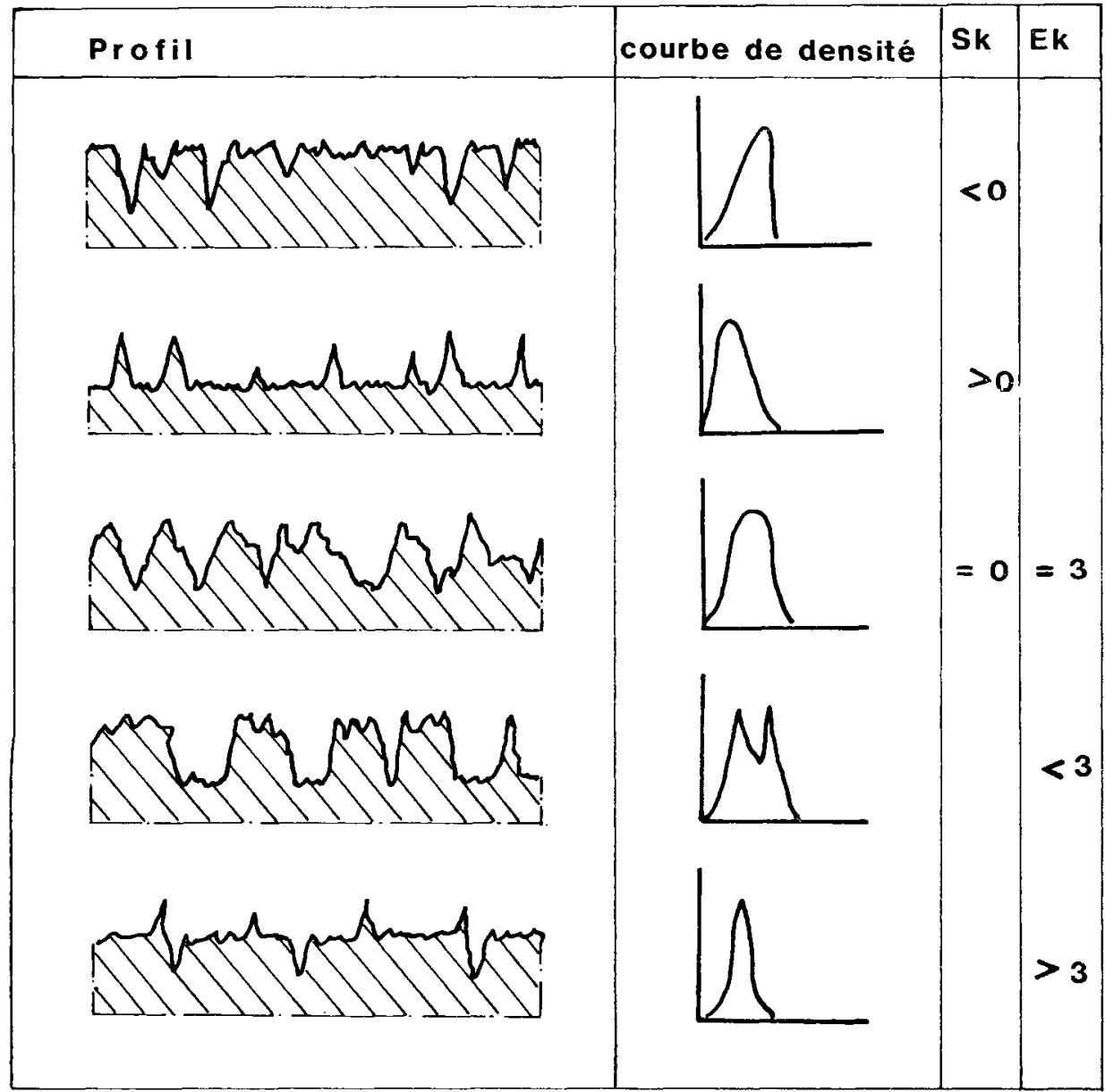


TABLEAU 2

Valeurs numériques caractéristiques de l'état de surface obtenues sur une feuille de placage tranché (chêne).

Numerical characteristic values of roughness on sliced oak veneer.

Les résultats ci-après portent sur les hauteurs $(979$ points)

Pas en abscisse des courbes ci-après $=1,154499$ microns

\begin{tabular}{|c|c|}
\hline Variance de la distribution des hauteurs & 23,94 \\
\hline Ecart type (RMS) $\ldots \ldots \ldots \ldots \ldots \ldots \ldots$ & 4.89 microns \\
\hline Coefficient d'asymétrie : skewness & $-0,68$ \\
\hline Coefficient d'aplatissement : curtosis & 5,29 \\
\hline Critères $R_{i l}$ & 3,37 microns \\
\hline
\end{tabular}

\subsection{Chemin d'évolution morphologique}

Lorsqu'une surface évolue au cours du temps, il est possible de suivre son évolution dans le plan $\left(\mathrm{S}_{\mathrm{k}}, \mathrm{E}_{\mathrm{k}}\right)$; on obtient alors le chemin d'évolution de la surface (FAyolle, 1983; Mathia, 1983); on peut, à partir de ce principe, envisager la délimitation des zones possibles pour un plan ligneux donné dans le plan $\left(\mathrm{S}_{\mathrm{k}}, \mathrm{E}_{\mathrm{k}}\right)$. On saura alors quelle est l'optimisation possible de la rugosité des surfaces pour chaque essence et le procédé d'élaboration qui s'y rapporte. On pourra suivre dans ce même plan, l'évolution des surfaces en fonction des paramètres humidité, température, temps, autre aspect vital pour la mise en œuvre optimale du matériau (problèmes de collage des bois verts, vieillissement, etc.).

\section{Conclusions}

La méthodologie proposée ici n'en est qu'à ses débuts; elle est susceptible d'amélioration et surtout adaptable à l'objectif que l'on est amené à se poser et aux phénomènes à mettre en évidence.

Le bois, matériau hétérogène et anisotrope, nécessite une méthode d'exploration suffisamment avancée et souple pour en définir correctement l'état de surface et les limites possibles. Le dispositif brièvement décrit dans ses grandes lignes et l'analyse qui en résulte pourront répondre à de nombreuses questions relatives à l'emploi possible du bois et aux problèmes de liaisons qualité-emploi.

D'autre part, à la lumière des quelques expériences menées, au niveau des déformations du bois lors de la prise des données par palpeur mécanique, il apparaît que le phénomène de pénétration en surface est loin d'être négligeable, et qu'il devrait être pris en compte dans l'interprétation de certains résultats, où les efforts exercés sur le palpeur sont trop importants en regard du diamètre de pointe de celui-ci (mesure de rétractibilité, mesure de diamètres de carottes, ...). 
Enfin, au niveau du capteur nécessairc au palpage des surfaces en bois, nous avons retenu les caractéristiques suivantes: diamètre de pointe du cône : 15 microns, charge appliquée : 0.003 Newtons, lincrément de déplacement du capteur étant fonction des capacités de traitement informatique des variables $Z(X, Y)$ et de l'ordre $\left(1^{\prime r}, 2^{\prime \prime}, 3^{\prime \prime}\right.$ et $\left.4^{\prime \prime}\right)$ des défauts que l'on veut mettre en évidence. Il $1 n^{\prime}=n$ demeure pas moins qu'il sera nécessaire de développer les expérimentations afin de définir les critères objectifs et universels d'états de surface utilisables sur le matériau bois.

\section{Summary \\ Reflexion on surfaces and measure of wood roughness}

The relationship between wood structural aspect and capacity for an utilization involve the measure of quality criterions of wood products.

Within this framework, we here a study on the measure of smoothness characteristic parameters. But, two of the specific problems of wood are structures heterogeneity and low density.

A measurement method with a stylus apparatus is proposed and also an interpretation of results obtained with this method.

Reçu le 23 juin 1983.

Accepté le 25 juillet 1983.

\section{Références bibliographiques}

AFNOR, 1972. Etats de surface des produits, prescriptions 1. Généralités, tcrminologic, définitions. NF $E$ 05.015.

C.T.B., 1973. Le comportement du bois au feu. Cahier 74 du Centre Technique du Bois, 3" édition.

Ebewele R.O., River B.H., Koutsky J.A., 1980. Tapered double cantilever beam fracture test of phenolic - wood adhesive joints.. Part. II - Effects of surface roughness, the nature of surface aging on joint fracture encrgy. Wood and Fiber, 12 (1), 40-65.

Fayolle C., 1983. Analyse statistique de la topographie des surfaces. Thèse de DoctcurIngćnieur, Ecole Centrale de Lyon.

Kikata Y., 1958. Studies on surface roughness of wood. II - A simple apparatus for determining surface roughness and its applicable conditions. J. Jap. Wood Res. Soc., vol. 4, n" 6, 216-219.

MAHCHaL R., 1983. Intérêts de la prise en compte de caractéristiques physiques et anatoniques simples du bois de hêtre mesurables sur des échantillons non destrictifs pour l'appréciation de la qualité des placages d'éhénisterie produtits à partir des mêmes individus. D.E.A. "Sciences du Bois», Nancy.

Mignot J., 1983. Analyse microtopographique des surfaces. Univ. Franche-Comté, I.U.T. Belfort.

Riout J., 1979. Les états de surface. Notes techniques CETIM N ${ }^{\text {os }} 18$ et 19.

Timoshenko S.P., 1968. Résistance des matériaux. Tome 2. Dunod Technique. 